\title{
Slow Cracklike Dynamics at the Onset of Frictional Sliding
}

\author{
Eran Bouchbinder, ${ }^{1}$ Efim A. Brener, ${ }^{1,2}$ Itay Barel, ${ }^{3}$ and Michael Urbakh ${ }^{3}$ \\ ${ }^{1}$ Chemical Physics Department, Weizmann Institute of Science, Rehovot 76100, Israel \\ ${ }^{2}$ Peter Grünberg Institut, Forschungszentrum Jülich, Jülich 52425 Germany \\ ${ }^{3}$ School of Chemistry, Tel Aviv University, Tel Aviv 69978, Israel \\ (Received 21 March 2011; published 28 November 2011)
}

\begin{abstract}
We propose a friction model which incorporates interfacial elasticity and whose steady state sliding relation is characterized by a generic nonmonotonic behavior, including both velocity weakening and strengthening branches. In 1D and upon the application of sideway loading, we demonstrate the existence of transient cracklike fronts whose velocity is independent of sound speed, which we propose to be analogous to the recently discovered slow interfacial rupture fronts. Most importantly, the properties of these transient inhomogeneously loaded fronts are determined by steady state front solutions at the minimum of the sliding friction law, implying the existence of a new velocity scale and a "forbidden gap" of rupture velocities. We highlight the role played by interfacial elasticity and supplement our analysis with 2D scaling arguments.
\end{abstract}

DOI: 10.1103/PhysRevLett.107.235501

The frictional strength and stability of spatially extended interfaces is important for a wide range of natural and man-made systems [1-3]. Yet, several fundamental aspects of it are not well understood. One of these is the onset of frictional sliding in which the (initially static) interface separating two bodies in frictional contact fails under shear forces, giving rise to relative shear motion.

This transition was recently observed to be mediated by the propagation of interfacial fronts [4-7]. By tracking the real contact area between two bodies, it was demonstrated that in addition to fast sub-Rayleigh and supershear cracklike modes $[4,7,8]$, there exist slow cracklike modes which travel at well-defined velocities significantly smaller than the Rayleigh wave speed, but, nevertheless, play a significant role in interface weakening. Furthermore, these experiments highlight the key role of inhomogeneity to frictional stability and mode selection [7], and the relevance of plastic deformation of interlocking asperities to frictional strength [6]. These important and unexplained observations, especially the nature and properties of the slow cracklike fronts, is our main focus.

In this Letter we develop a friction model based on the dynamics of microcontacts at frictional interfaces. We demonstrate the existence of a frictional instability prior to the onset of sliding, which excites cracklike fronts whose velocity is independent of sound speed. Most importantly, we show that the properties of these slow fronts propagating under transient inhomogeneous conditions is determined by steady state front solutions at the minimum of the sliding friction law, where velocity-weakening behavior crosses over to a velocity-strengthening one [2,9]. Our work is strongly influenced by recent important numerical investigations of a microscopic 1D model [10] and macroscopic 2D analytic results $[11,12]$.
PACS numbers: 62.20.Qp, 46.32.+x, 46.50.+a, 91.55.Fg

We consider an elastic body with a rough surface that is being pushed along a frictional rigid substrate. Focus first on the shear traction $\sigma_{x y}$ along the interface and write the following expression:

$$
\sigma_{x y}(x, y=0, t)=\bar{\sigma}(x, t)+\eta \partial_{t} u_{x}(x, y=0, t),
$$

where $y=0$ is the location of the interface and $x$ is the position along it. Here $\bar{\sigma}$ is associated with interfacial contact dynamics, $\eta$ is a viscous-friction coefficient (whose dimensions are stress $\times$ time $\times$ length $^{-1}$ ), and $u_{x}$ is the displacement jump (slip) across the sliding interface. We choose the velocity-strengthening part $\eta \partial_{t} u_{x}$ to have the simplest possible form, which does not affect the generality of our results, as long as such a branch exists. Since the substrate is assumed rigid, we set $u_{y}(x, y=0, t)=0$.

A rough contacting interface, i.e., one which is not atomically flat, is characterized by an evolving real contact area (to be distinguished from the nominal one which is typically much larger) and an associated traction $\bar{\sigma}$. Therefore, the next step is to write down dynamic equations for $\bar{\sigma}$ and $A$, the ratio between the real contact area and the nominal one. To address the former, we consider an interfacial boundary layer of size $h$ (the effective height of the interface) whose deformation is quantified by $\partial_{t} u_{x} / h$. In the absence of irreversible slip and when $A$ is a constant, we expect $\partial_{t} u_{x} / h=\partial_{t} \bar{\sigma} /\left(A \mu_{0}\right)$, where $\mu_{0}$ is the interfacial elastic modulus. This immediately leads to an elastic response in the form $\bar{\sigma}=\mu_{0} A u_{x} / h$, which is expected on theoretical grounds. Both the linear relation between $\bar{\sigma}$ and $u_{x}$, and the linear dependence on $A$, were experimentally demonstrated in [13]. This interfacial elasticity, in spite of its potential importance, is typically not included in standard friction laws [2] (but note [14,15]).

The onset of irreversible slip processes is characterized by the existence of a threshold above which $\partial_{t} u_{x} / h$ 
contains an additional contribution that we write as $\theta\left(\left|\sigma_{x y}\right| / A-\sigma_{c}\right) \bar{\sigma} \partial_{t} u_{x} /\left(A \mu_{0} D\right)$, where $\sigma_{c}$ is the shear strength or yield stress of the microcontacts. Note that following standard rate-and-state friction models, we set the time scale of evolution here to be proportional to a typical geometric length $D$ (the microcontacts size) over the slip rate, i.e., to the contact lifetime [2]. Furthermore, note that in the $\theta$ function, the macroscopic traction $\sigma_{x y}$ is enhanced at the microcontacts level by a factor $A^{-1} \gg 1$. Collecting the two additive contributions to $\partial_{t} u_{x} / h$ and isolating $\partial_{t} \bar{\sigma}$, we obtain

$$
\partial_{t} \bar{\sigma}=\mu_{0} A \partial_{t} u_{x} / h-\theta\left(\left|\sigma_{x y}\right| / A-\sigma_{c}\right) \bar{\sigma} \partial_{t} u_{x} / D .
$$

The fact that $D$ plays the role of a phenomenological decay length of $\bar{\sigma}$ during sliding becomes evident when both sides of Eq. (2) are divided by $A \partial u_{x}$ and $\partial t$ is eliminated. Finally, we note that Eq. (2) can be interpreted in analogy with an elastic-plastic bulk constitutive relation.

The dynamics of $A$, which is expressed as

$$
\partial_{t} A=\left(A_{0}-A\right) / \tau_{0}-\theta\left(\left|\sigma_{x y}\right| / A-\sigma_{c}\right) \kappa A \partial_{t} u_{x} / D,
$$

consists of two contributions; the first tends to increase $A$ to a limiting value $A_{0} \ll 1$ on a time scale $\tau_{0}$. In general $A_{0}$ depends on the normal stress and may "age" logarithmically on long time scales, but here it is constant. The second contribution accounts for the reduction of $A$ due to irreversible processes (plastic deformation and eventually fracture) and is similar to the corresponding term in Eq. (2). The dimensionless parameter $\kappa$ accounts for the possibility that $A$ decays on a time scale somewhat different than $\bar{\sigma}$, as suggested in [6]. Equations (1)-(3) constitute a "minimal" continuum friction model based on the detachment and reattachment dynamics of interfacial contacts. A closely related discrete model has been recently proposed in [10]. We stress that all of the quantities in Eqs. (1)-(3) are of interfacial nature and should be distinguished from their bulk counterparts.

By defining $\tilde{t}=t / \tau_{0}, \tilde{x}=x / D, \tilde{u}=u / D, \tilde{A}=A / A_{0}$, $\tilde{\sigma}_{x y}=\sigma_{x y} / \sigma_{c} A_{0}$, and $\tilde{\tilde{\sigma}}=\bar{\sigma} / \sigma_{c} A_{0}$, Eqs. (3) and (2) become

$$
\begin{gathered}
\partial_{\tilde{t}} \tilde{A}=(1-\tilde{A})-\theta\left(\left|\tilde{\sigma}_{x y}\right| / \tilde{A}-1\right) \kappa \tilde{A}\left|\partial_{\tilde{t}} \tilde{u}_{x}\right|, \\
\partial_{\tilde{t}} \tilde{\bar{\sigma}}=\alpha \tilde{A} \partial_{\tilde{t}} \tilde{u}_{x}-\theta\left(\left|\tilde{\sigma}_{x y}\right| / \tilde{A}-1\right) \tilde{\bar{\sigma}}\left|\partial_{\tilde{t}} \tilde{u}_{x}\right|,
\end{gathered}
$$

with $\alpha=\mu_{0} D / \sigma_{c} h$. Finally, we rewrite Eq. (1) as $\tilde{\sigma}_{x y}=$ $\tilde{\tilde{\sigma}}+\tilde{\eta} \partial_{\tilde{t}} \tilde{u}_{x}$, with $\tilde{\eta}=\eta D / \sigma_{c} \tau_{0} A_{0}$, which completes the derivation of our dimensionless friction law.

Macroscopic frictional phenomena intrinsically involve the coupling between bulk elastic deformation and the dissipative dynamics of the interface. Therefore, we should couple our friction law to a linear-elastic bulk of height $H$. The bulk is described by Lamé equation for the displacement field $\boldsymbol{u}(x, y, t)$ [16]. $\sigma_{x y}$ in Eq. (1) is a boundary condition at $y=0$ [recall that $u_{y}(x, 0, t)=0$ ]. Here we consider an elastic plate that is pushed along a frictional rigid surface, assuming that the shear strain in the plate is always small except in the vicinity of the contacting interface. In this limit (corresponding to small $H$ ), $\sigma_{x y}$ is no longer a boundary condition but rather a term in a $1 \mathrm{D}$ equation, and $u_{x}(x, y, t)$ can be replaced by $u_{x}(x, y=0, t)$. This leads to [17]

$$
\tilde{\rho} \partial_{\tilde{t} \tilde{t}} \tilde{u}_{x}=\tilde{\mu} \partial_{\tilde{x} \tilde{x}} \tilde{u}_{x}+\tilde{\sigma}_{d}-\tilde{\bar{\sigma}}-\tilde{\eta} \partial_{\tilde{t}} \tilde{u}_{x} .
$$

Here $\tilde{\rho}=\rho H D / \sigma_{c} \tau_{0}^{2} A_{0}$ and $\tilde{\mu}=\mu H / \sigma_{c} D A_{0}$, where $\rho$ is the mass density and $\mu$ is the bulk shear modulus [17]. $\tilde{\sigma}_{d}$ is the dimensionless external driving stress.

In order to mimic the experimental edge-loading setup of [4-7], we study Eqs. (4)-(6) for $\tilde{x} \geq 0$ with a localized driving force of the form $\tilde{\sigma}_{d}(\tilde{x}, \tilde{t})=\tilde{K}_{d}\left[\tilde{v}_{d} \tilde{t}-\right.$ $\left.\tilde{u}_{x}(0, \tilde{t})\right] \delta(\tilde{x})$. Here $\tilde{K}_{d}$ and $\tilde{v}_{d}$ are the rescaled driving spring constant and velocity, respectively, and $\delta(\cdot)$ is a $\delta$ function.

We first studied our model numerically [18]. A typical solution is presented in Fig. 1. Initially, the response is purely elastic; i.e., $\tilde{A}=1$ in panel (b) and the driving force rises almost linearly in panel (a). At $\tilde{t}^{*}(\simeq 25$ here), a cracklike front is initiated at the trailing edge $(\tilde{x}=0)$, leaving behind a reduced contact area $\tilde{A}<1$. This cracklike front is accompanied by a relatively low slip rate, see panel (d), but by a significant stress transfer resulting in an inhomogeneous stress state, see panel (c). Finally, this front induces a small, but noticeable, drop in the driving force, see the early time deviation from the straight dashed line in panel (a).

Later, at $\tilde{\hat{t}}(\simeq 110)$, an instability in which a short-lived cracklike front is initiated at the trailing edge and arrests at a finite distance (smaller than the system's length $\tilde{L}$ ) occurs. The front's velocity is much higher than the one initiated at $\tilde{t}^{*}$ (compare the slopes in panel (b) and recall

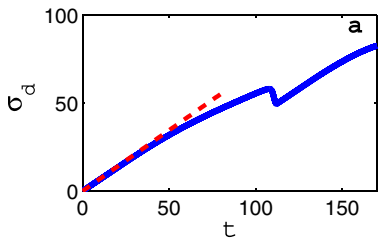

$\bar{\sigma}(x, t)$
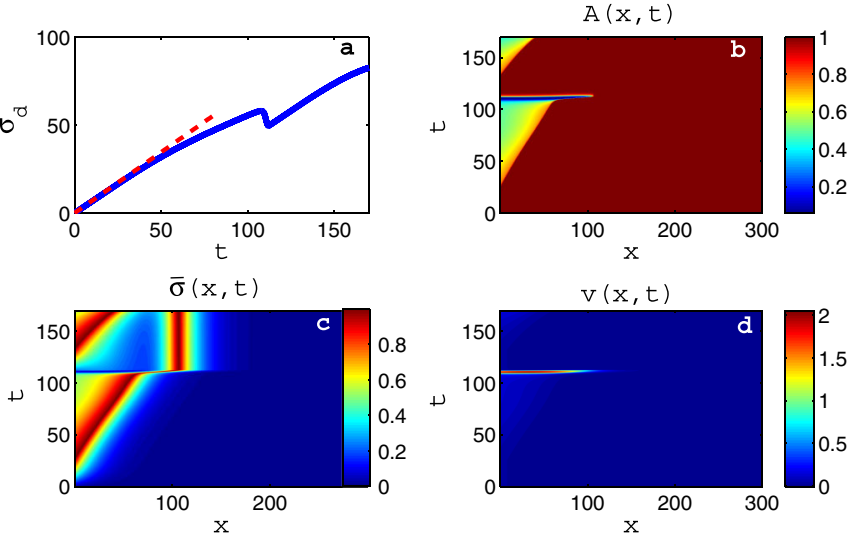

FIG. 1 (color online). A solution of Eqs. (4)-(6) with $\tilde{\rho}=0.01$, $\tilde{\mu}=300, \kappa=8, \alpha=1.05, \tilde{\eta}=0.1, \tilde{K}_{d}=2.5$, and $\tilde{v}_{d}=0.3$. (a) The driving force $\tilde{\sigma}_{d}(t)$. (b) The contact area $\tilde{A}(x, t)$. (c) $\tilde{\bar{\sigma}}(\tilde{x}, \tilde{t})$. (d) The slip velocity $\tilde{v}(\tilde{x}, \tilde{t})=\partial_{\tilde{t}} \tilde{u}_{x}(\tilde{x}, \tilde{t})$. For simplicity, the tildes are omitted from the axis labels. 
that the smaller the slope, the higher the velocity) and is accompanied by a sharp increase in the slip rate, see panel (d). Finally, the transient front transfers a highly concentrated stress distribution to the interior of the material, see panel (c), and the jump in slip rate at the edge causes an abrupt drop in the driving force, see panel (a).

After the transient front arrests, the contact area recovers to its background level (in general this will not be the case since normal stress variations, which are absent here, will carry memory of past deformation), see panel (b), but a highly concentrated stress distribution remains inside the material, as a signature of the irreversible slip that took place, see panel (c). Another front, similar to the one initiated at $\tilde{t}^{*}$, is initiated at the trailing edge soon after $(\tilde{t} \simeq 130)$. It eventually triggers another instability that results in a transient front that propagates deeper into the material (not shown). This complex precursory activity repeats itself until a front reaches the leading edge $(\tilde{x}=\tilde{L}$ ), whereupon macroscopic sliding occurs (not shown), in agreement with experimental observations [5]. We focus on the first precursory event.

In order to develop a theoretical understanding of the cracklike dynamics that precedes the onset of sliding, we first focus on the elastic response at $\tilde{t}<\tilde{t}^{*}$. In this regime, the contact area remains intact, $\tilde{A}=1$, and the $\theta$ function in Eqs. (4) and (5) vanishes. Then, for $\tilde{t}<\tilde{t}^{*}$, we obtain

$$
\tilde{u}_{x}(\tilde{x}, \tilde{t})=\tilde{K}_{d} \tilde{v}_{d} \tilde{t} \exp (-\sqrt{\alpha / \tilde{\mu}} \tilde{x}) \theta(\tilde{x}) /\left(\tilde{K}_{d}+\sqrt{\alpha \tilde{\mu}}\right)
$$

where the inertial term, which is negligible in this regime, was omitted. This solution is valid until $\tilde{\tilde{\sigma}} / \tilde{A}$ first equals unity (note that $\tilde{\eta} \partial_{\tilde{t}} \tilde{u}_{x}$ is negligible in this regime, thus we can replace $\sigma_{x y}$ with $\left.\bar{\sigma}\right)$. This happens when $\tilde{\bar{\sigma}}\left(0, \tilde{t}^{*}\right)=$ $\alpha \tilde{u}_{x}\left(0, \tilde{t}^{*}\right)=1$, i.e., $\tilde{t}^{*}=\left(\tilde{K}_{d}+\sqrt{\alpha \tilde{\mu}}\right) /\left(\alpha \tilde{K}_{d} \tilde{v}_{d}\right)$. The exponential spatial variation in Eq. (7) is the 1D Green's function (whose analog at higher dimensions is a powerlaw distribution) and hence it characterizes any purely elastic region, even at times $\tilde{t}>\tilde{t}^{*}$.

We proceed to discuss the cracklike front that initiates at $\tilde{t}^{*}$. To understand its nature, we adopt a fracture mechanics perspective [16], which tells us that our edgeloading system is intrinsically stable. That is, the stress level near the tip of a crack is a decreasing function of the length of the crack under constant loading at the trailing edge. That means that the tip region, which has to satisfy some fracture criterion (in our case $\tilde{\sigma}_{x y} / \tilde{A}=1$ ), cannot propagate unless the external loading is increased. This implies that the dimensionless velocity $\bar{c}$ of such a crack must satisfy $\bar{c} \propto \tilde{v}_{d}$. The prediction $\bar{c} \propto \tilde{v}_{d}$ is fully supported by the numerical solutions. The fact that fracture mechanics predicts a stable front is important since it immediately suggests that the instability taking place at $\tilde{\hat{t}}$ has a frictional origin, absent in tensile fracture.

The front that initiates at $\tilde{t}^{*}$ plays an important role in triggering the frictional instability at $\tilde{\hat{t}}$. In particular, it is responsible for the transfer of stress from the trailing edge to the interior of the material and hence for the buildup of an inhomogeneous stress distribution. Such inhomogeneous stress distributions were shown to play an important role in selecting various interfacial cracklike modes in [7]. The frictional instability at $\tilde{\hat{t}}$ is accompanied by a transient cracklike front whose dimensionless velocity $\tilde{c} \gg \bar{c}$. Understanding the nature and properties of this front is a major goal of this Letter.

We do not consider here the onset (nucleation) of instability, which we observed to involve fast, Rayleigh-like, contact area disturbance initiated at the trailing edge, but rather focus on the emerging short-lived cracklike fronts. In order to develop a theoretical understanding of these fronts we should discuss some basic properties of our friction law. Consider the spatially homogeneous fixed points of Eqs. (4) and (5) as a function of the slip rate $\tilde{v}=\partial_{\tilde{t}} \tilde{u}_{x}$. At $\tilde{v}=0$ there exist only elastic solutions with $0 \leq \tilde{\sigma}_{x y}<1$. For $\tilde{v}>0$, we find the following sliding solutions:

$$
\tilde{\sigma}_{x y}(\tilde{v})=\alpha /(1+\kappa \tilde{v})+\tilde{\eta} \tilde{v} \quad \text { for } \tilde{v}>0
$$

Since $\tilde{\sigma}_{x y} \geq \tilde{A}$ for sliding solutions (the $\theta$ function equals unity), internal consistency demands that $\alpha \geq 1$. An important feature of the sliding friction law in Eq. (8) is its nonmonotonic behavior stressed above, where $\tilde{\sigma}_{x y}(\tilde{v})$ attains a minimum at $\tilde{v}_{m}$, with a finite value $\tilde{\sigma}_{d}^{c}=\tilde{\sigma}_{x y}\left(\tilde{v}_{m}\right)$. The complete steady state sliding friction law is shown in Fig. 2 (left). The parameters $\alpha$ and $\kappa$, control the rate of velocity-weakening for $0<\tilde{v} \lesssim \tilde{v}_{m}$, while $\tilde{\eta}$ controls the rate of velocity strengthening for $\tilde{\boldsymbol{v}} \gtrsim \tilde{\boldsymbol{v}}_{m}$. Their relative values determine the crossover at $\tilde{v}=\tilde{v}_{m}$. We suggest that this nonmonotonic behavior is generic [2,9].

It is instructive to consider also situations in which the homogeneous loading $\tilde{\sigma}_{d}$, rather than the slip rate $\tilde{v}$, is controlled. In this case, for $\tilde{\sigma}_{d}<\tilde{\sigma}_{d}^{c}$ (see the dash-dotted line in Fig. 2), we have only one solution (with $\tilde{v}=0$ ), which is a stable solution. At $\tilde{\sigma}_{d}=\tilde{\sigma}_{d}^{c}$ (see the dashed line in Fig. 2) another solution emerges at $\tilde{v}_{m}$ (marked by a
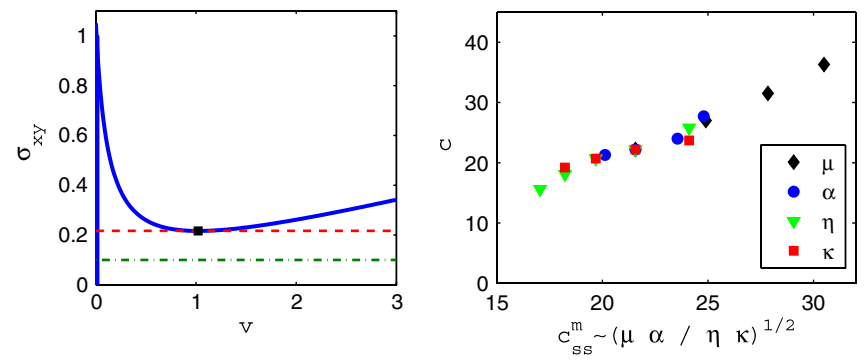

FIG. 2 (color online). Left: The steady friction law $\tilde{\sigma}_{x y}(\tilde{\boldsymbol{v}})$ (parameters as in Fig. 1) (solid line). The horizontal lines correspond to homogeneous loading. Right: The transient front velocity $\tilde{c}$ vs the minimal steady state velocity $\tilde{c}_{s s}^{m} \sim \sqrt{\tilde{\mu} \alpha / \tilde{\eta} \kappa}$. The tildes are omitted from all quantities. 
filled square in Fig. 2). This allows for the propagation of a steady state "phase transition" front in which a sliding domain invades a jammed one. For $\tilde{\sigma}_{d}>\tilde{\sigma}_{d}^{c}$ we have two finite $\tilde{v}$ solutions, one with $0<\tilde{v}<\tilde{v}_{m}$ in the velocityweakening (unstable) regime and one with $\tilde{v}>\tilde{v}_{m}$ in the velocity-strengthening (stable) regime, which also allow for steady state front solutions.

Can one relate the steady state homogeneously loaded fronts to the transient fronts observed under inhomogeneous loading? Our basic idea is that the transient fronts are short-lived excitations of fronts corresponding to the minimum of the sliding friction law. To test this idea, denote the minimal velocity of steady state fronts by $\tilde{c}_{s s}^{m}$, set $\partial_{\tilde{t}}=$ $-\tilde{c}_{s s}^{m} \partial_{\tilde{x}}$ in Eqs. (4)-(6), and note that Eq. (8) implies $\tilde{v}_{m} \sim \sqrt{\alpha / \tilde{\eta} \kappa}$ and $\tilde{\sigma}_{d}^{c} \sim \tilde{\eta} \tilde{v}_{m}$, for $\kappa \gg 1$. Hence, the scaling version of Eqs. (5) is $\tilde{c}_{s s}^{m} / \tilde{\ell}_{\bar{\sigma}} \sim \tilde{v}_{m}$ and of Eq. (6):

$$
\tilde{\mu} \tilde{v}_{m} / \tilde{\ell}_{\bar{\sigma}} \tilde{c}_{s s}^{m}+\tilde{\sigma}_{d}^{c}-\tilde{\bar{\sigma}}-\tilde{\eta} \tilde{v}_{m}=0 \Rightarrow \tilde{\mu} \tilde{v}_{m} \sim \tilde{\ell}_{\bar{\sigma}} \tilde{c}_{s s}^{m} .
$$

Here $\tilde{\ell}_{\bar{\sigma}}$ is spatial scale of variation of $\tilde{\bar{\sigma}}, \tilde{\sigma}_{d}^{c} \sim \tilde{\eta} \tilde{v}_{m}$ and $\tilde{\bar{\sigma}} \simeq 1$ (near the front edge, where the $\theta$ function first equals unity) were used and inertia was neglected. Solving for $\tilde{c}_{s s}^{m}$ and $\tilde{\ell}_{\bar{\sigma}}$, we obtain (with $\tilde{H}=H / D$ )

$$
\tilde{c}_{s s}^{m} / \tilde{s} \sim \sqrt{\tilde{H} / \tilde{\ell}_{\kappa}} \Rightarrow \tilde{c}_{s s}^{m} \sim \sqrt{\tilde{\mu} \alpha / \tilde{\eta} \kappa}
$$

and $\tilde{\ell}_{\bar{\sigma}} \sim \sqrt{\tilde{H} \tilde{\ell}} . \tilde{\ell}=\tilde{\mu} / \tilde{H}$ and $\tilde{\ell}_{\kappa}=\tilde{\mu} \kappa / \tilde{H} \tilde{\eta} \alpha>\tilde{\ell}$ are two rescaled friction-related length scales, and $\tilde{s}=\tilde{\mu} / \tilde{H} \tilde{\eta}$.

Equation (10) contains an elastic bulk property $\tilde{\mu}$ and the three friction parameters $\alpha, \tilde{\eta}, \kappa$. Adopting our idea that $\tilde{c} \sim \tilde{c}_{s s}^{m}$, it provides an analytic prediction for the transient front velocity $\tilde{c}$. We measured $\tilde{c}$ directly from numerical solutions. In Fig. 2 (right) we show $\tilde{c}$ vs the minimal steady state velocity $\tilde{c}_{s s}^{m} \sim \sqrt{\tilde{\mu} \alpha / \tilde{\eta} \kappa}$. Different symbols denote variations of each parameter, shown in the legend, while the other three were held fixed. The result is striking: the data points collapse on a single linear curve with a slope of order unity. This result provides strong evidence in favor of our basic idea; the properties of the transient inhomogeneously loaded fronts are indeed determined by the steady state front solutions at the minimum of the sliding friction law.

A crucial point to note is that Eq. (10) suggests that $\tilde{c}$ is independent of the sound speed, $\tilde{c}_{s}=\sqrt{\tilde{\mu} / \tilde{\rho}}$. For example, in Fig. 1, $\tilde{c} \simeq 20$ while $\tilde{c}_{s} \simeq 170$; i.e., $\tilde{c}$ and the sound speed are separated by an order of magnitude. Instead of $\tilde{\rho}$ a combination of friction parameters determine $\tilde{c}$. In particular, we highlight the fact that $\tilde{c}$ depends explicitly on the interfacial elastic modulus $\mu_{0} \sim \alpha$. It is important to note that the spectrum of front solutions starts at a finite value $\tilde{c}_{s s}^{m}$ and hence there exists a "forbidden gap" of velocities below it, in contrast to ordinary tensile cracks (or standard "phase transition fronts"). This is consistent with the experiments in $[4,7]$, where the slow front is always characterized by a well-defined finite velocity. Therefore, we propose that the transient fronts observed here are analogous to the slow fronts discovered recently $[4,7]$ and whose velocity should be described by $c_{s s}^{m}$.

What about sub-Rayleigh or supershear fronts? We suspect that this is a matter of the mechanical conditions at nucleation. When the stored energy levels are low, as in the examples considered above, $\tilde{c}_{s s}^{m}$ may be the most relevant "attractor" for the dynamics. On the other hand, when the stored energy levels at nucleation are higher, a continuous spectrum of solutions with $\tilde{c}>\tilde{c}_{s s}^{m}$ may be excited. This seems perfectly consistent with the findings of $[7,19]$, where mode selection is shown to be controlled by the conditions at nucleation.

While we have not yet numerically studied our model in 2D and hence at the moment cannot comment on the validity of our main idea in $2 \mathrm{D}$, we can show that $\tilde{c}_{s s}^{m}$ varies smoothly from $1 \mathrm{D}$ to $2 \mathrm{D}$ as $\tilde{H}$ is increased and hence it is qualitatively independent of dimensionality. To see this we note that the $1 \mathrm{D}$ limit is valid for $\tilde{H} \ll \tilde{\ell}_{\kappa}, \tilde{\ell}$. Consider then the $2 \mathrm{D}$ regime $\tilde{\ell} \ll \tilde{H}$. The distinguishing feature of dimensions higher than 1 is the existence of (cracklike) power-law singularity at $\tilde{\ell} \ll|\tilde{x}| \ll \tilde{H}$. One can show that in the singular region the boundary conditions of the present model reduce to those of $[11,12]$, who found that $\tilde{v} \sim|\tilde{x}|^{(-1+\epsilon) / 2}$, where $\tan (\pi \epsilon / 2) \sim \tilde{c}_{s s}^{m} / \tilde{s}$.

If we assume $\tilde{H} \gg \tilde{\ell}_{\kappa}, \tilde{\ell}$, then our result is identical to Eq. (11) in [11], which here reads $\epsilon \sim \ln \left(\tilde{H} / \tilde{\ell}_{\kappa}\right) / \ln (\tilde{H} / \tilde{\ell})$. If we assume $\tilde{\ell} \ll \tilde{H} \ll \tilde{\ell}_{\kappa}$, we can still use the relation $\tilde{c}_{s s}^{m} / \tilde{\ell}_{\bar{\sigma}} \sim \tilde{v}_{m}$, but now with $\tilde{\ell}_{\bar{\sigma}}$ replaced by $\tilde{\ell}$ and $\tilde{v}_{m}$ by $\tilde{v}_{m}(\tilde{H} / \tilde{\ell})^{(1-\epsilon) / 2}$, where the latter describes the increase of the slip rate on a scale $\tilde{\ell}$ (it decays back to $\tilde{v}_{m}$ on a scale $\tilde{H} \gg \tilde{\ell}$ ). The resulting expression is $\tilde{c}_{s s}^{m} / \tilde{s} \sim$ $\sqrt{\tilde{H} / \tilde{\ell}}(\tilde{\ell} / \tilde{H})^{\epsilon / 2}$. It matches the $1 \mathrm{D}$ result of Eq. (10), when $\tilde{H} \sim \tilde{\ell}$, and the $2 \mathrm{D}$ result stated above, when $\tilde{H} \sim \tilde{\ell}_{\kappa}$. We reiterate that the most important implication of this $2 \mathrm{D}$ scaling analysis is that the presence of a cracklike singularity does not change the qualitative properties of the spectrum of steady state front velocities, which still starts at a finite value.

We believe our results derive from robust properties of dry friction and hence are general (and not model specific). In future work we plan to test our ideas in 2D numerical simulations, to include the effect of normal stress variations and aging on the contact area, to address the full spectrum of cracklike fronts and to make quantitative comparison to experimental data.

We thank O. Ben-David and J. Fineberg for numerous insightful discussions. E. B. acknowledges support of the James S. McDonnell Foundation, the Harold Perlman Family Foundation, and the Robert Rees Applied Research Fund. E. A. B. acknowledges support of the Erna and Jacob Michael visiting professorship funds at Weizmann Institute of Science. M.U. acknowledges support by the Israel 
Science Foundation, Grant 1109/09 and by the GermanIsraeli Project Cooperation Program (DIP).

[1] M. Urbakh et al., Nature (London) 430, 525 (2004).

[2] T. Baumberger and C. Caroli, Adv. Phys. 55, 279 (2006).

[3] Y. Ben-Zion, Rev. Geophys. 46, RG4006 (2008).

[4] S. M. Rubinstein, G. Cohen, and J. Fineberg, Nature (London) 430, 1005 (2004).

[5] S. M. Rubinstein, G. Cohen, and J. Fineberg, Phys. Rev. Lett. 98, 226103 (2007).

[6] O. Ben-David, S. M. Rubinstein, and J. Fineberg, Nature (London) 463, 76 (2010).

[7] O. Ben-David, G. Cohen, and J. Fineberg, Science 330, 211 (2010).

[8] K. Xia, A. Rosakis, and H. Kanamori, Science 303, 1859 (2004).

[9] Z. Reches and D. A. Lockner, Nature (London) 467, 452 (2010).
[10] O. M. Braun, I. Barel, and M. Urbakh, Phys. Rev. Lett. 103, 194301 (2009).

[11] E. A. Brener and V. I. Marchenko, JETP Lett. 76, 211 (2002).

[12] E. A. Brener, S. V. Malinin, and V.I. Marchenko, Eur. Phys. J. E 17, 101 (2005).

[13] P. Berthoud and T. Baumberger, Proc. R. Soc. A 454, 1615 (1998).

[14] G. L. Povirk and A. Needleman, J. Eng. Mater. Technol. 115, 286 (1993).

[15] Z. Shi, A. Needleman, and Y. Ben-Zion, Int. J. Fract. 162, 51 (2010).

[16] B. R. Lawn, Fracture of Brittle Solids (Cambridge University Press, Cambridge, U.K., 1993).

[17] See Supplemental Material at http://link.aps.org/ supplemental/10.1103/PhysRevLett.107.235501 for explicit expressions.

[18] We used $\sigma_{x y} \simeq \bar{\sigma}$ in the $\theta$ function, which has a minor effect on the results.

[19] Z. Shi, Y. Ben-Zion, and A. Needleman, J. Mech. Phys. Solids 56, 5 (2008). 